\title{
Article \\ Nutrition and Vitamin Deficiencies Are Common in Orthopaedic Trauma Patients
}

\author{
Jordan E. Handcox ${ }^{1}$, Jose M. Gutierrez-Naranjo ${ }^{1} \mathbb{D}$, Luis M. Salazar ${ }^{2} \mathbb{D}$, Travis S. Bullock ${ }^{1}$, Leah P. Griffin ${ }^{3}$ \\ and Boris A. Zelle ${ }^{1, *}$ \\ 1 Department of Orthopaedics, UT Health San Antonio, 7703 Floyd Curl Dr., San Antonio, TX 78229, USA; \\ handcox@uthscsa.edu (J.E.H.); gutierreznar@uthscsa.edu (J.M.G.-N.); bullockt@uthscsa.edu (T.S.B.) \\ 2 Long School of Medicine, UT Health San Antonio, 7703 Floyd Curl Dr., San Antonio, TX 78229, USA; \\ salazarlm@livemail.uthscsa.edu \\ 3 Medical Solutions Division, 3M Health Care, San Antonio, TX 78249, USA; lpgriffin@mmm.com \\ * Correspondence: zelle@uthscsa.edu; Tel.: +1-210-743-4102
}

Citation: Handcox, J.E.;

Gutierrez-Naranjo, J.M.; Salazar, L.M.; Bullock, T.S.; Griffin, L.P.; Zelle, B.A. Nutrition and Vitamin Deficiencies Are Common in Orthopaedic Trauma Patients. J. Clin. Med. 2021, 10, 5012. https://doi.org/10.3390/jcm10215012

Academic Editor: Roman Pfeifer

Received: 5 October 2021

Accepted: 26 October 2021

Published: 28 October 2021

Publisher's Note: MDPI stays neutral with regard to jurisdictional claims in published maps and institutional affiliations.

Copyright: (c) 2021 by the authors. Licensee MDPI, Basel, Switzerland. This article is an open access article distributed under the terms and conditions of the Creative Commons Attribution (CC BY) license (https:// creativecommons.org/licenses/by/ $4.0 /)$.

\begin{abstract}
Macro- and micronutrients play important roles in the biological wound-healing pathway. Although deficiencies may potentially affect orthopaedic trauma patient outcomes, data on nutritional deficiencies in orthopaedic trauma patients remain limited in the literature. The purpose of this study was to (1) evaluate the prevalence of macro- and micronutrient deficiencies in orthopaedic trauma patients with lower extremity fractures and (2) evaluate the impact of such deficiencies on surgical site complications. This retrospective study identified 867 patients with lower extremity fractures treated with surgical fixation from 2019 to 2020. Data recorded included albumin, prealbumin, protein, vitamins $\mathrm{A} / \mathrm{C} / \mathrm{D}$, magnesium, phosphorus, transferrin and zinc, as well as wound complications. Nutritional deficiencies were found for prealbumin, albumin and transferrin at $50.5 \%, 23.4 \%$ and $48.5 \%$, respectively. Furthermore, a high prevalence of micronutrient deficiencies (vitamin A, 35.4\%; vitamin C, 54.4\%; vitamin D, 75.4\%; and zinc, 56.5\%) was observed. We also recorded a statistically significant difference in wound complications in patients who were deficient in prealbumin $(21.6 \% \mathrm{vs}$. $6.6 \%, p=0.0142)$ and vitamin C (56.8\% vs. $28.6 \%, p=0.0236)$. Our study outlines the prevalence of nutritional deficiencies in an orthopaedic trauma population and identifies areas for possible targeted supplementation to decrease wound complications.
\end{abstract}

Keywords: orthopaedic trauma; nutritional deficiencies; vitamins; lower extremity; wound complications; nutrition wound healing

\section{Introduction}

The important role of nutrition in wound healing has been well documented in the literature, with macro- and micronutrients considered vital at every step of the wound healing pathway [1-3]. Unfortunately, malnutrition is common worldwide [4] and can be from a variety of causes, including advanced age, disease-related, food-insecurity/hunger or a mismatch between caloric intake and quality of nutrients consumed [5]. Malnutrition is a known contributor to poor clinical outcomes, from increased morbidity and mortality to wound and surgical complications [6,7]. As such, there is much interest in evaluating the role of nutrition in orthopaedic trauma patients, a vulnerable population sensitive to the effects of malnutrition.

Previous literature has demonstrated that malnutrition, as defined by hypoalbuminemia, is common in the orthopaedic trauma population [8]. Moreover, these authors recorded hypoalbuminemia and obesity as predictors of wound complications. Additionally, albumin deficiencies have been shown to correlate with wound complications in patients undergoing joint replacement surgery [9] and readmission rates for patients undergoing elective spine surgeries [10]. However, prior research has mostly focused on 
the geriatric populations, elective orthopaedic surgeries, and albumin and prealbumin as serum markers for malnutrition [11-14].

Even fewer studies have looked at the prevalence of micronutrient deficiencies in orthopaedic trauma patients. Among elderly hospitalized patients, vitamins C and D are commonly deficient $[15,16]$; however, there is limited data on its prevalence among a younger trauma population. Severe micronutrient deficiencies have well-known consequences, such as severe vitamin D deficiency leading to rickets and osteoporosis. Subtle deficiencies below the reference range may lead to wound-healing complications and other lesser-known sequela [5], and the orthopaedic literature has just started to explore the relationship between micronutrient deficiencies and negative clinical outcomes. For example, it has been shown that zinc deficiencies may lead to wound-healing complications in patients undergoing hemiarthroplasty [17]. Also, vitamin D has been shown to impact fracture healing rates in orthopaedic trauma patients [18]. Yet, data on micronutrient deficiencies in the orthopaedic trauma population remain limited in the literature.

The purpose of this study is to (1) evaluate the prevalence of macro- and micronutrient deficiencies in orthopaedic trauma patients with lower extremity fractures and (2) evaluate the impact of such deficiencies on surgical site complications in patients with high-risk lower extremity fractures. Our hypothesis is that deficiencies are common in the orthopaedic trauma population, and these deficiencies may be associated with an increase in surgical site complications in high-risk lower extremity fractures.

\section{Materials and Methods}

This study is a retrospective database analysis of orthopaedic trauma patients undergoing surgical fixation of their lower extremity fractures treated at a university-based level 1 trauma center between the years of 2019 and 2020. The study protocol was approved by our Institutional Review Board, and data collection, methods and analysis were performed in accordance with their rules and regulations. Inclusion criteria were patients over 18 years old with a minimum of 3 months follow-up and lower extremity fractures, identified through our electronic medical record system using the coding database. Patients were identified using the OTA classification system to include femur, tibia, tibia/fibula, fibula, talus, calcaneus and foot fractures. Subjects were excluded if they were under 18 years old, mentally or cognitively impaired, prisoners, or if they presented with a pathologic fracture, as well as those with less than 3 months follow-up.

Demographic data included age, gender, race/ethnicity, BMI, and the American Society of Anesthesiologists scale [19], as well as mechanism of injury and closed versus open injury. As our primary outcome measure, we recorded the available laboratory data on patients, including both macro- and micronutrient data: albumin (3.2-5.0 g/dL), prealbumin (17.0-37.1 mg/dL), protein total serum (6.2-8.1 g/dL), albumin/globulin ratio (1.06-1.61), transferrin (206-382 mg/dL), vitamin A (0.30-1.20 mg/L), vitamin C (23-114 mmol/L), vitamin D (30-80 ng/mL), vitamin K (0.22-4.88 nmol/L), magnesium (1.6-2.2 mg/dL), phosphorus $(2.4 / 4.6 \mathrm{mg} / \mathrm{dL})$, zinc $(60.0-120 \mu \mathrm{g} / \mathrm{dL})$, selenium $(23-190 \mu \mathrm{g} / \mathrm{L})$, TSH $(0.350-5.500 \mu \mathrm{IU} / \mathrm{mL})$ and PTH $(19-88 \mathrm{pg} / \mathrm{mL})$. An expanded panel of micronutrient data was obtained for patients who were deemed high risk by their treating orthopaedic surgeon; this expanded lab draw was at the discretion of the surgeon.

Secondary clinical outcome measures were tracked through a review of inpatient and outpatient charts and included data on wound complications (surgical site infections, wound dehiscence, hematoma) and surgical complications (malunion, nonunion, symptomatic hardware).

The statistical analysis was performed using SAS software version 9.4 (SAS Institute Inc., Cary, NC, USA). Categorical variables are summarized as count and percent. Chisquared and Fisher's exact test were used to calculate differences for categorical variables. All tests were conducted at the alpha level of 0.05 . 


\section{Results}

\subsection{Patient-Level Demographic Data}

We identified 867 patients who met the inclusion criteria. Of these patients, $28.7 \%$ were age 65 or older. There were slightly more male patients (56.9\%) compared to female patients. A majority of patients identified as White (95.6\%) or Hispanic/Latino (62.1\%). Finally, nearly $40 \%$ of patients were obese, as defined by BMI $\geq 30$ (Table 1 ).

Table 1. Demographic and clinical data.

\begin{tabular}{cc}
\hline Number of Patients & $n=867$ \\
\hline Age $\geq 65$ & $249(28.7 \%)$ \\
Ethnicity-Hispanic & $538(62.1 \%)$ \\
Race-White & $829(95.6 \%)$ \\
Gender (\% Male) & $493(56.9 \%)$ \\
BMI $\geq 30$ & $343(39.6 \%)$ \\
Fracture location & $n=1008$ \\
Proximal Femur & $195(19.4 \%)$ \\
Femoral Shaft & $107(10.6 \%)$ \\
Distal Femur & $34(3.4 \%)$ \\
Proximal Tibia & $98(9.7 \%)$ \\
Tibial Shaft & $84(8.3 \%)$ \\
Ankle/Pilon & $352(34.9 \%)$ \\
Talus & $20(2.0 \%)$ \\
Calcaneus & $51(5.1 \%)$ \\
Foot & $17(1.7 \%)$ \\
Others & $38(3.8 \%)$ \\
Infection, Non-traumatic & $12(1.2 \%)$ \\
\hline
\end{tabular}

\subsection{Nutritional Deficiencies}

Albumin was measured for 745 patients, and of these, $23.4 \%$ were malnourished, as defined by albumin $<3.5 \mathrm{~g} / \mathrm{dL}$. Approximately half of the patients were deficient in prealbumin $(50.5 \%)$. Finally, nearly half of the patients $(48.5 \%)$ were deficient in transferrin.

Of those patients who had micronutrient data measured, $35.4 \%$ were deficient in vitamin $A, 54.4 \%$ were deficient in vitamin $C$, and $75 \%$ were deficient in vitamin D. Over half of the patients were deficient in zinc ( $56.5 \%$ of patients). We did not observe significant deficiencies in magnesium, selenium or vitamin K (Table 2).

Table 2. Nutritional deficiencies by macro- or micronutrient.

\begin{tabular}{ccc}
\hline Nutritional Markers & $N$ & Deficient \\
\hline Prealbumin & 99 & $50(50.5 \%)$ \\
Albumin & 745 & $174(23.4 \%)$ \\
Protein total serum & 735 & $62(8.4 \%)$ \\
Albumin/globulin ratio & 734 & $512(69.8 \%)$ \\
Transferrin & 68 & $33(48.5 \%)$ \\
Vitamin A & 82 & $29(35.4 \%)$ \\
Vitamin C & 57 & $31(54.4 \%)$ \\
Vitamin D & 215 & $162(75.4 \%)$ \\
Vitamin K & 42 & $1(2.4 \%)$ \\
Magnesium & 619 & $21(3.4 \%)$ \\
Phosphorus & 609 & $100(16.4 \%)$ \\
Zinc & 92 & $52(56.5 \%)$ \\
Selenium & 63 & $0(0 \%)$ \\
Thyroid-stimulating hormone & 154 & $9(5.8 \%)$ \\
Parathyroid hormone & 97 & $2(2.1 \%)$ \\
\hline
\end{tabular}




\subsection{Nutritional Deficiencies by Demographic Group}

There were significant differences in nutritional deficiencies between demographic groups. Of the patients 65 and older, $83.3 \%$ were deficient in prealbumin, compared to only $46.0 \%$ of patients younger than 65 years old $(p=0.0153)$. A similar trend was recorded with age and albumin, where $37 \%$ of patients over 65 years old were deficient, compared to $17.7 \%$ of patients under 65 years old $(p<0.0001)$. Compared to younger patients, patients over 65 years old were also at increased risk of deficiency in serum protein $(11.7 \%$ deficient versus $7.1 \%, p=0.0396)$ and transferrin $(81.8 \%$ deficient versus $42.1 \%, p=0.0158)$. With regards to age-related differences in micronutrients, patients over 65 years old were not at increased risk of deficiency in vitamins A, C, or zinc. Additionally, advanced age was protective against vitamin D deficiency, as $84.7 \%$ of younger patients had vitamin $\mathrm{D}$ deficiency, compared to $59.0 \%$ of patients over $65(p<0.0001)$.

Females were more likely to be deficient in albumin $(29.2 \%)$ compared to males $(19.2 \%$, $p=0.0015)$. Micronutrient data showed that Hispanic patients were more likely to be vitamin D deficient than non-Hispanic patients $(82.4 \%$ versus $65.6 \%, p=0.0047)$. We did not observe statistically significant differences between these demographic groups in the remaining serum markers.

\subsection{Complications by Nutritional Deficiency}

To measure the rate of wound complications, we assessed the data at the fracture level, identifying 1008 individual lower extremity fractures, 181 (18.0\%) of which had a wound complication. Low prealbumin was associated with a statistically significant difference in wound complications. We found that $21.6 \%$ of fractures with a prealbumin deficiency had a wound complication, compared to $6.6 \%$ of those with normal prealbumin levels $(p=0.0142)$. Vitamin C deficiency was also associated with wound complications; where $56.8 \%$ sustained a wound complication, compared to only $28.6 \%$ with normal vitamin C levels $(p=0.0236)$.

\section{Discussion}

Identifying macro- and micronutrient deficiencies in our orthopaedic trauma population is important, as it allows us to (1) understand the prevalence of this issue and (2) perform targeted interventions, which may lead to improvement in outcomes. Our data suggest that nutritional deficiencies have a high prevalence in orthopaedic trauma patients. Furthermore, macro- and micronutrient deficiencies may be associated with wound complications, most notably prealbumin and vitamin $C$ deficiency.

There were some limitations to our study. One limitation was its retrospective design; this resulted in limited data for some of the studied micronutrients. Also, expanded micronutrient data collection was at the discretion of the treating surgeon at the time of hospital presentation. This led to variability in which nutritional markers were drawn for each patient. This may have contributed to the underrepresentation of certain micronutrients and difficulties in identifying other surgical complications, such as malunion/nonunion. Also, these data are from our local orthopaedic trauma population, which may lead to geographic variation in deficiency patterns.

Previous studies demonstrated improved outcomes with nutritional supplements in the geriatric population $[14,20]$ and enhanced callus formation with zinc supplementation in young adult trauma patients with lower extremity fractures [21]. To our knowledge, there have not been any data reported on nutritional deficiencies using multiple serum markers in orthopaedic trauma patients with injuries at high risk for infection.

We have demonstrated profound malnutrition rates, including a hypoalbuminemia rate of $23.4 \%$, which is slightly lower than previously found in this population $(39.4 \%$, Egbert et al.). Over half of our patients were deficient in prealbumin, which more closely correlates with perioperative nutritional deficiency, given its shorter half-life [22]. Prealbumin deficiency has previously been shown to correlate with surgical site infections in patients undergoing spinal surgery [23], and we did confirm a statistically significant 
difference in wound complications among those who were prealbumin deficient in our population. Almost half of the patients (48.5\%) were deficient in transferrin, which also indicates significant malnutrition [24] and has been implicated in wound complications in arthroplasty [25]. Regarding micronutrient deficiencies, we found substantial deficiencies in vitamins $\mathrm{A}, \mathrm{C}, \mathrm{D}$, and zinc, which is in line with the data available in the geriatric population $[11,14]$. In addition, Hispanic patients were also much more likely to be vitamin $\mathrm{D}$ deficient. Finally, we demonstrated that vitamin $\mathrm{C}$ deficiency is common, as is consistent with the existing literature [15] and found that deficiency in vitamin C may lead to wound complications.

Our study reinforces prior literature on the prevalence and impact of hypoalbuminemia in an orthopaedic trauma population $[8,26]$ and confirms that malnutrition is a risk factor for wound complications $[27,28]$. Our study also confirms prior research demonstrating significant vitamin D deficiency in a diverse trauma population [12]. Our novel data on micronutrient deficiencies in the orthopaedic trauma population provides preliminary evidence for vitamin/nutritional supplementation in the perioperative period for a younger orthopaedic trauma population in order to improve clinical outcomes.

\section{Conclusions}

In summary, our study demonstrates a high prevalence of macro- and micronutrient deficiencies in an orthopaedic trauma patient population with lower extremity fractures. Deficiencies in prealbumin, and vitamins C, D and zinc were common, with over half of patients in our study group proving to be deficient. We also identified demographic risk factors for malnutrition, such as age, sex and ethnicity. Finally, we demonstrated that prealbumin and vitamin $\mathrm{C}$ may be associated with wound complications. This study lays the groundwork for identifying targeted supplement and nutritional interventions that may reduce the risk of surgical site complications. The reversal of these deficiencies in the perioperative period has the potential to improve patient outcomes and reduce hospital costs.

Author Contributions: Conceptualization, J.E.H., J.M.G.-N., L.M.S., T.S.B., L.P.G. and B.A.Z.; data curation, J.M.G.-N., L.M.S. and T.S.B.; formal analysis, L.P.G.; funding acquisition, L.P.G. and B.A.Z.; investigation, J.M.G.-N., L.M.S. and T.S.B.; methodology, J.E.H., J.M.G.-N., L.M.S., T.S.B., L.P.G. and B.A.Z.; project administration, J.E.H. and B.A.Z.; resources, B.A.Z.; software, L.P.G.; supervision, B.A.Z.; validation, L.P.G.; visualization, J.E.H.; writing-original draft, J.E.H., J.M.G.-N., L.M.S., T.S.B., L.P.G. and B.A.Z.; writing-review and editing, J.E.H., J.M.G.-N., L.M.S., T.S.B., L.P.G. and B.A.Z. All authors have read and agreed to the published version of the manuscript.

Funding: This research was partially funded by $3 \mathrm{M}$ KCI Inc.

Institutional Review Board Statement: The study was conducted according to the guidelines of the Declaration of Helsinki and approved by the Institutional Review Board of the University of Texas Health Science Center at San Antonio (project ID 165197).

Informed Consent Statement: Patient consent was waived by the IRB of our institution, as our study does not include any specific interventions or patient contact.

Data Availability Statement: The data are not publicly available online.

Conflicts of Interest: The authors declare no conflict of interest.

\section{References}

1. Palmieri, B.; Vadalà, M.; Laurino, C. Nutrition in wound healing: Investigation of the molecular mechanisms, a narrative review. J. Wound Care 2019, 28, 683-693. [CrossRef]

2. Stechmiller, J.K. Understanding the Role of Nutrition and Wound Healing. Nutr. Clin. Pract. 2010, 25, 61-68. [CrossRef]

3. Wild, T.; Rahbarnia, A.; Kellner, M.; Sobotka, L.; Eberlein, T. Basics in nutrition and wound healing. Nutrition 2010, 26, 862-866. [CrossRef] [PubMed]

4. World Health Organization. Malnutrition. Available online: https://www.who.int./news-room/fact-sheets/detail/malnutrition (accessed on 18 October 2021). 
5. Cederholm, T.; Barazzoni, R.; Austin, P.; Ballmer, P.; Biolo, G.; Bischoff, S.C.; Compher, C.; Correia, I.; Higashiguchi, T.; Holst, M.; et al. ESPEN guidelines on definitions and terminology of clinical nutrition. Clin. Nutr. 2017, 36, 49-64. [CrossRef]

6. Reber, E.; Gomes, F.; Vasiloglou, M.F.; Schuetz, P.; Stanga, Z. Nutritional Risk Screening and Assessment. J. Clin. Med. 2019, 8, 1065. [CrossRef] [PubMed]

7. Tulchinsky, T.H. Micronutrient deficiency conditions: Global health issues. Public Health Rev. 2010, 32, 243-255. [CrossRef]

8. $\quad$ Egbert, R.C.; Bouck, T.T.; Gupte, N.N.; Pena, M.M.; Dang, K.H.; Ornell, S.S.; Zelle, B.A. Hypoalbuminemia and Obesity in Orthopaedic Trauma Patients: Body Mass Index a Significant Predictor of Surgical Site Complications. Sci. Rep. 2020, $10,1953$. [CrossRef]

9. Gu, A.; Malahias, M.; Strigelli, V.; Nocon, A.A.; Sculco, T.P.; Sculco, P.K. Preoperative Malnutrition Negatively Correlates with Postoperative Wound Complications and Infection After Total Joint Arthroplasty: A Systematic Review and Meta-Analysis. J. Arthroplast. 2019, 34, 1013-1024. [CrossRef] [PubMed]

10. Adogwa, O.; Elsamadicy, A.A.; Mehta, A.; Cheng, J.; Bagley, C.A.; Karikari, I.O. Preoperative Nutritional Status Is an Independent Predictor of 30-Day Hospital Readmission after Elective Spine Surgery. Spine 2016, 16, S271. [CrossRef] [PubMed]

11. Ernst, A.; Wilson, J.M.; Ahn, J.; Shapiro, M.; Schenker, M.L. Malnutrition and the Orthopaedic Trauma Patient: A Systematic Review of the Literature. J. Orthop. Trauma 2018, 32, 491-499. [CrossRef] [PubMed]

12. Zellner, B.; Dawson, J.; Reichel, L.; Schaefer, K.; Britt, J.; Hillin, C.; Reitman, C. Prospective Nutritional Analysis of a Diverse Trauma Population Demonstrates Substantial Hypovitaminosis D. J. Orthop. Trauma 2014, 28, e210-e215. [CrossRef]

13. Bohl, D.; Shen, M.; Hannon, C.; Fillingham, Y.; Darrith, B.; Della Valle, C. Serum Albumin Predicts Survival and Postoperative Course Following Surgery for Geriatric Hip Fracture. J. Bone Jt. Surg. Am. 2017, 99, 2110-2118. [CrossRef]

14. Roberts, J.L.; Drissi, H. Advances and Promises of Nutritional Influences on Natural Bone Repair. J. Orthop. Res. 2020, 38, 695-707. [CrossRef] [PubMed]

15. Teixeira, A.; Carrié, A.S.; Généreau, T.; Herson, S.; Cherin, P. Vitamin C deficiency in elderly hospitalized patients. Am. J. Med. 2001, 111, 502. [CrossRef]

16. Boettger, S.F.; Angersbach, B.; Klimek, C.N.; Wanderley, A.L.M.; Shaibekov, A.; Sieske, L.; Wang, B.; Zuchowski, M.; Wirth, R.; Pourhassan, M. Prevalence and predictors of vitamin D-deficiency in frail older hospitalized patients. BMC Geriatr. 2018, 18, 219. [CrossRef]

17. Zorrilla, P.; Salido, J.A.; Lopez-Alonso, A.; Silva, A. Serum Zinc as a Prognostic Tool for Wound Healing in Hip Hemiarthroplasty. Clin. Orthop. Relat. Res. 2004, 420, 304-308. [CrossRef] [PubMed]

18. Pourfeizi, H.H.; Tabriz, A.; Elmi, A.; Aslani, H. Prevalence of vitamin D deficiency and secondary hyperparathyroidism in nonunion of traumatic fractures. Acta Med. Iran. 2013, 51, 705-710. [PubMed]

19. Daabiss, M. American Society of Anaesthesiologists physical status classification. Indian J. Anaesth. 2011, 55, 111-115. [CrossRef] [PubMed]

20. He, Y.; Xiao, J.; Shi, Z.; He, J.; Li, T. Supplementation of enteral nutritional powder decreases surgical site infection, prosthetic joint infection, and readmission after hip arthroplasty in geriatric femoral neck fracture with hypoalbuminemia. J. Orthop. Surg. Res. 2019, 14, 292. [CrossRef]

21. Sadighi, A.; Roshan, M.M.; Moradi, A.; Ostadrahimi, A. The effects of zinc supplementation on serum zinc, alkaline phosphatase activity and fracture healing of bones. Saudi Med. J. 2008, 29, 1276-1279.

22. Keller, U. Nutritional Laboratory Markers in Malnutrition. J. Clin. Med. 2019, 8, 775. [CrossRef]

23. Tempel, Z.; Grandhi, R.; Maserati, M.; Panczykowski, D.; Ochoa, J.; Russavage, J.; Okonkwo, D. Prealbumin as a serum biomarker of impaired perioperative nutritional status and risk for surgical site infection after spine surgery. J. Neurol. Surg. Part A Cent. Eur. Neurosurg. 2015, 76, 139-143.

24. Gariballa, S.; Forster, S. Effects of acute-phase response on nutritional status and clinical outcome of hospitalized patients. Nutrition 2006, 22, 750-757. [CrossRef]

25. Roche, M.; Law, T.Y.; Kurowicki, J.; Sodhi, N.; Rosas, S.; Elson, L.; Summers, S.; Sabeh, K.; Mont, M.A. Albumin, Prealbumin, and Transferrin May Be Predictive of Wound Complications following Total Knee Arthroplasty. J. Knee Surg. 2018, 31, $946-951$. [CrossRef]

26. Wilson, J.; Lunati, M.; Grabel, Z.; Staley, C.; Schwartz, A.; Schenker, M. Hypoalbuminemia Is an Independent Risk Factor for 30-Day Mortality, Postoperative Complications, Readmission, and Reoperation in the Operative Lower Extremity Orthopaedic Trauma Patient. J. Orthop. Trauma 2019, 33, 284-291. [CrossRef] [PubMed]

27. He, Z.; Zhou, K.; Tang, K.; Quan, Z.; Liu, S.; Su, B. Perioperative hypoalbuminemia is a risk factor for wound complications following posterior lumbar interbody fusion. J. Orthop. Surg. Res. 2020, 15, 538. [CrossRef] [PubMed]

28. Cross, M.; Yi, P.; Thomas, C.; Garcia, J.; Della Valle, C. Evaluation of Malnutrition in Orthopaedic Surgery. JAAOS 2014, 22, 193-199. [CrossRef] [PubMed] 